\title{
Establishing the Relationship between Personality Traits and Stress in an Intelligent Environment
}

\author{
Marco Gomes, Tiago Oliveira, Fábio Silva, Davide Carneiro and Paulo Novais \\ Department of Informatics, University of Minho \\ \{marcogomes, toliveira, fsilva, dcarneiro,pjon\}@di.uminho.pt
}

\begin{abstract}
Personality traits play a key role in the shaping of emotions, moods, cognitions, and behaviours of individuals interacting in a virtual environment. The personalities one exhibits reflect one's perception of the world and are demonstrated in the act of communication. Thus, the evaluation of a message can be changed due to stress and mood variations. Being able to identify the degree of relationship between one's personality characteristics and one's current stress state can thus facilitate the communication process. In particular, in this paper it is studied the correlation between some personality traits and the stress levels exhibited by users' interactions. To do so a novel approach was followed in which an intelligent environment is used to support the stress recognition process providing important personality related information. An experiment has been designed for the purpose of addressing the estimation of relevant aspects of interactions that occur in a rich sensory environment. Outputs from the experiment, such as the relation between personality characteristics and stress, can be used to maximize the benefits of virtual environments and its applications in fields such as learning, medicine or conflict resolution.
\end{abstract}

Keywords: Intelligent Environment, Stress, Emotions, Personality

\section{Introduction}

As a society we nowadays experience a sense of proximity that stems from the unprecedented development in communication technologies over the last two decades. It is possible to communicate instantly with virtually anyone in the globe, leading to the feeling of a so-called "Global Village". However, this proximity is shallow and artificial as new communication mechanisms become increasingly poor, lacking many of the traditional Human aspects of communication. Addressing this subject, the lack of the non-verbal elements between interlocutors limits successful communication in Virtual Environments (VEs). In that sense, communicating in the absence of this information can be difficult [1]. To address it, preponderant factors in human communication must be covered, such as stress and personality traits. The use of a combination of multiple Artificial Intelligence techniques and, more particularly Ambient Intelligence techniques, can help to fill this gap.

Different personality types process and communicate information differently. Each personality type has a different impact in an individual's response to stress. Stress influences not only the efficiency of the communication but also the quality of its perception 
or even the relationship between the individuals communicating. Emotions significantly influence our social skills, our motivation and our actions in general. Likewise, the emotional state of one's interlocutor is very important to understand the true meaning of their words. Considering that people are individuals and behave individually, once you have identified the personalities and their communicative behaviours, an intelligent environment can potentiate more efficient communication mechanisms. The approach followed looks at how individual's behaviours change under stress and emotional stimuli. Specifically, we study the interaction of individuals with devices commonly used to communicate in virtual settings: keyboard and mouse. Preliminary work allowed to identify features extracted from interaction with these devices that are significantly affected by stress [2]. This allows us to identify and study behavioural changes associated to specific states of an individual such as stress, fatigue or emotional arousal. To accomplish this, one must understand how the patterns of interaction, the relationship between personality traits and stress can affect the human communication. Knowing how to deal with these dimensions will can improve our communication with others and our understanding of ourselves.

\section{Multimodal Approach to Stress and Personality Dimensions}

In this section, we look at the multi-dimensionality of stress and personality. Indeed, given the multiplicity of factors that influence human experience and the nature of this work, a single-modality approach to measure the effects of stress and personality would not be suited. Therefore, we propose the use of a multi-modal one. Indeed, it can be stated that human behaviour can be understood as an all-encompassing spectrum of what people do including thinking and feeling, which are influenced, for example, by culture, attitudes or emotions. Moreover, it is commonly accepted that people are all different from each other because they have different personalities, they are inside specific cultures, and they have distinct individual histories.

All behaviour can be considered as situational, i.e., when the situation or event changes, the behaviour changes too: the intention to carry out a certain action or the fashion in which we d it can be changed by the present circumstances. In the context of an intelligent environment, environmental factors can have a significant influence on shaping individual factors (e.g., personality types, cognitive maturity, emotional status and social experiences) and play a significant role in one's life. Human behaviour exhibits how a human being reacts intrinsically (determined by personality) and externally (stress responses) facing the stimuli that occurs in their environment.

Concerning stress, a consensual definition is still an open discussion in the scientific community. The main reasons for this lie in its multi-dimensionality and subjective nature, which led to multiple interpretations. And, with so many factors that can contribute to stress, it can be rather difficult to define it. Scientists have circumvented this problem by defining it empirically. To this end, researchers start to focus upon cognitive and behavioural consequences of stress, and stress became viewed as a mind-body, psychosomatic, or psycho-physiologic phenomenon. An interpretation of this phenomenon could refer stress as a psycho-physiologic arousal response occurring in the body as result of stimuli, and these stimuli becomes a stressor by virtue of the cognitive inter- 
pretation of the individual. This interpretation of stress is used in this paper to analyse stress in virtual environment scenarios.

In what concerns personality, it determines how a person reacts intrinsically to certain events. In a certain way, it can be seen like a long-term emotion, because it is formulated in the early years of life and remains almost unchanged during lifetime. The Big-Five Personality (BFP) theory also called Five Factor Model (FFM)[3] is a common definition of personality traits that indicate that is possible to separate them into five properties. Although psychological and physiological adverse ambient conditions can produce significantly changes in a person, the authors steer this topic to a very specific set of variables: sound, temperature and luminosity are studied as external factors that affect well-being and mood states.

Other researchers have also researched and debated the influence of such variables in the impact of mood change in people [10]. In a noisy environment people have diverging sensitivity to the volume of the sound. For instance, people that are sensitive to sound have a predisposition to become more anxious when the volume rises. Others, if provoked, can even become aggressive or hostile. In hot places psychological changes occur if the temperature increases, such as irritability, fatigue and discomfort. Very high values can also provoke hostility or violent attitudes. Assessing this type of information from the environment allows to estimate and even foresee the kind of emotional reactions that may arise, through a classification of mood states.

Different emotional reactions can be deduced in part by the personality structure of each person, represented in this study by the BFP theory and a mood simulator reacting to environment configurations. Taking into consideration the aforementioned stress and personality dimensions, we follow a multi-modal approach to analyse how they are related with each other.

\section{An Intelligent Environment to Enhance Emotional and Stress Recognition}

One of the main characteristics of Intelligent Environments is the key role that the user plays as the focus of the entire process. In other words, the process starts by collecting data about the user and the environment in which the user is situated, and it finishes by acting intelligently for the benefit of the user. Therefore, an intelligent environment with diverse devices and functionalities was built, aiming to compile and provide information about the user's context and state to the applications being used. This environment is composed of hard sensors (i.e., hardware sensors, in the traditional sense) and soft sensors (i.e., software sensors such as sensors for the interaction with the mouse and the keyboard), independent systems (with logic and intelligent services) and an integration framework.

The two most interesting components, from the point of view of this paper and described ahead in more detail, are the Stress Recognition and the Mood Recognition modules. The first classifies, in real-time, the level of stress of a user; the second provides an outlook of the user's mood using information about the personality traits previously provided. Based on this, a behavioural and contextual analysis is performed, applying Artificial Intelligence techniques in order to detect patterns and relationships 
among the selected features (see Sec. 4). A more detailed description of each of these modules is provided in the two following sub-sections.

\subsection{Stress Recognition Module}

The quantification of human stress can improve the way people behave and communicate in a virtual environment. Having this information, a system may correctly monitor how each issue or event is affecting each particular individual. The developed Stress Recognition Module is based in previous work [2] and relies on an approach of Behavioural Biometrics: a field of science that seeks to study the particularities of the behaviour of each individual in a given situation [13]. It can be used, for example, to accurately determine the identity of people for access control, through their behaviour (e.g. how they talk, how they walk, how they use the computer).

This field is very closed to the more traditional field of Biometrics, that relies on unique physical characteristics (e.g. hand size, fingerprint), independent of human behaviour but unique for each individual. However, Behavioural Biometrics looks at behavioural characteristics (e.g. the typing rhythm on a keyboard, the way the user looks at the screen of the computer). These characteristics change with factors such as mood, fatigue, stress or others [6]. When one knows how each individual behaves in each scenario or condition, one can then identify the individual from their behaviours or from changes in these behaviours. This is the key idea behind Behavioural Biometrics.

For this study Keystroke Dynamics and Mouse Dynamics were selected to this end. From between more than two dozen features that can be extracted in a non-invasive and transparent way, the following were selected from these two types of behavioural biometrics:

- Time between Keys (TBK): time spent between the use of two keys, that is, the time between events $K E Y \_U P$ and $K E Y \perp D O W N$ row. Unit: millisecond

- Key Down Time (KDT) - time spent since the key is pressed down and is released later, in other words, time since the event KEY DOWN and KEY_UP consecutively. Unit: milliseconds

- Absolute Sum of Angles (ASA) - absolute sum of the angles when turning left or right pointer during its travel. This measure tries to find just how much the mouse "turned", regardless of the direction to which it turned. Unit: degree

- Mouse Velocity $(M V)$ - Mouse Velocity - velocity at which the cursor travels. The distance travelled by the mouse (in pixels) between a $C 1$ coordinate $(x 1, y 1)$ and $C 2(x 2, y 2)$ corresponding to time 1 and time 2 over travel time (in milliseconds). Unit: Pixel/Milliseconds

- Mouse Acceleration (MA): acceleration of the mouse at a given time. The acceleration value is calculated using the mouse velocity on movement time. Unit: Pixel/Milliseconds

- Distance Pointer To Line Between Clicks (DPTLBC) - between each two consecutive clicks, measures the distance between all the points of the path travelled by the mouse and the closest point in a straight line (that represents the shortest path) between the coordinates of the two clicks. 


\subsection{Mood Recognition Module}

Personality traits, beliefs, mood or the state of the physical environment have influence on the emotional arousal of people. In a computational system it is still a challenging process to acquire accurate and rich information in this domain but there are some approaches proposed in the research community that have had broad acceptance. In regard to the representation of a personality this work uses an OCEAN structure similar to the approach adopted by the ALMA framework to represent the personality of people and initiate mood states [5]. In this theory personality is defined by a set of variables:

$$
\begin{aligned}
& \text { personality }(o, c, e, a, n) \\
& \text { let } o, c, e, a, n \in[-1,1]
\end{aligned}
$$

These $o, c, e, a, n$ variables represent five personality traits which mean Openness, Conscientiousness, Extraversion, Agreeableness and Neuroticism, respectively. The process of acquiring personality traits from real people is carried out through their answers to questionnaires used by psychologists in psychological studies. In this particular case it was used the summarized version of the Newcastle Personality Assessor (NPA) as a reference questionnaire [8].

Contrary to the personality, which is regarded as almost static during people's lifetimes, mood is a temporary state of the human mind, can last for minutes, hours or even days. It is seen as medium-term emotion. Given that personality also influences mood, the preliminary definition of personality traits, initial mood assessments can be implemented considering the predominant mood state for each personality. Individual personality parameters can also be taken into consideration for mood updates over time for each person.

The mood recognition module here described is responsible for assessing the current mood of each person using their personality structure and the perception of environmental variables such as sound, temperature and luminosity. Mood representation is based on the PAD space computationally designed by Gehbard in his work ALMA [5]. A person's mood is represented using the three variables that define PAD space, $P, A$ and $D$ for actual pleasure, arousal and dominance respectively. These variables are in the domain $[-1,1]$ to create the representation of the eight moods that are possible: hostile, exuberant, disdainful, docile, bored, anxious, dependent and relaxed. Other variables included on Mood are the emotionalWeight to represent the weight of the emotions related with each personality and const which represents the velocity of the Mood update.

Everyone is different and, just as literature suggests, people with high trend of neuroticism $(n)$ have more probability to be emotionally negative i.e., they have predisposition to feel emotions like hate, anger or distress more often than people with low score of this trend. In other hand extraversion (e) awakens positive feelings more often, such as joy, love or gratitude.

As a consequence, the calculation of the weight is carried out through the signal of emotion: if it is higher than emotionalWeight $=(e+1.0) *$ const else emotionalWeight $=$ $(n+1.0) *$ const. This variable is in the range $[0,2]$ turning emotionalWeight positive 
to facilitate future calculations. The constant const is used to reduce or increase the velocity when mood is crossing from state to state. In this work the value used was 0.1 . One of Mood's parametrized constructors receives as parameters personalities' values $o, c, e, a, n$. To initiate mood in relation to personality in the PAD space an weighted reference was used as in:

$$
\begin{aligned}
& P=0.59 a+0.19 n+0,21 e \\
& A=-0.59 n+0.30 a+0,15 o \\
& D=0.60 e-0.32 a+0.25 o+0.17 c \\
& \text { leto, }, e, a, \in[-1,1]
\end{aligned}
$$

This creates an initial mood state different from user to user, giving them a mood foundation that allows software agents to deal with different emotion types during the life time. These variables represent the mood state relating it with emotions triggered by events. The events are triggered by the environment's sound, luminosity and temperature conditions according to which the final mood is calculated. This final mood state is the predicted value for the exposure of people to those environment conditions after an infinite amount of time. The transition between mood states is assessed by applying an increment in the mood variables in the order and direction of the predicted final mood state after an event is triggered. An expression that makes the update of the current value with the displacement times the weight of emotion is used to calculate the displacement vector for each portion of time, as in :

$$
\begin{array}{r}
\overrightarrow{M_{\text {actual }} M_{\text {final }}}=M_{\text {final }}-M_{\text {actual }} \\
M_{\text {actual }}=M_{\text {actual }}+\frac{\overrightarrow{M_{\text {actual }} M_{\text {final }}}}{\text { emotionalWeight }}
\end{array}
$$

The vector $\overrightarrow{M_{\text {actual }} M_{\text {final }}}$ is divided in small pieces that are relative to emotionalWeight and this causes mood state to move more or less slowly, depending on each person's personality, over the time, thus preventing the instant passage from one state to another which does not happen in real life frequently.

Emotions are mapped in the PAD space using the OCC model [9] for emotion representation and ergonomic studies on the effect of the state temperature, luminosity and sound in emotion states [10]. From these studies and the current assessment of environment state, a final mood state can be calculated mapping the emotion derived and the current mood state using the PAD space and equation 2 . The equation assures that the mood state does not react instantly to new emotions but it is gradually affected by them.

\section{Practical Experiment}

As stated before, the main objective of this research work was to identify which and how human psychological traits are correlated to stress and how they can be pointed out. To demonstrate the relationship discussed in the previous sections, an experiment was set up in which we tried to estimate all the relevant aspects of the interaction between the 
individual and their environment that occur in a rich sensory environment (where the contextual modalities were monitored). This environment was equipped with sensors and devices that acquire different kinds of information from the user in a non-intrusive way (using the multimodal approach described in Sec. 2) ${ }^{1}$. To have a psychological profile of the individual under study we have chosen a small questionnaire, the Newcastle Personality Assessor (NPA), referenced in scientific literature.

The participants of the proposed experiment were volunteers from our institution. Eight individuals participated, both female and male, aged between 20 and 32. All these individuals were familiar with the devices used. Therefore, the interaction with them was not an obstacle. The first step of the experiment was to ask the volunteers to fill in the NPA individual questionnaire. The following step was the monitoring of the individuals' interaction with their devices (where the stress recognition module was installed), within the set environment. While the user conscientiously interacts with the system, a parallel and transparent process takes place in which the contextual and behavioural data is sent in a synchronized way to the platform (a framework deliberately designed to handle with stress and mood recognition). The platform, upon converting the sensory data into useful information, allows for a contextualized analysis of the users' data.

In previous studies, the features mentioned in Sec. 3.1 were used in successful attempts to detect the stress of an individual or, to be more precise, to identify the transition of an individual to a higher state of arousal [11]. Considering the hypothesis that the occurrence of stressed states may be related with some personality traits, it could be possible to establish an association between the features tested in the experiment and the answers collected from the NPA questionnaire. Since the objective is to classify the personality traits of an individual according to his interaction patterns with a machine, a special type of Bayesian Networks was used, the Naïve Bayes [7]. It is a simpler probabilistic model that assumes strong independence between the evidence variables. In the following section a more detailed description will be provided on the construction of said classifiers.

\subsection{Dataset Construction, Pre-Processing and Attribute Selection}

In order to construct the Naïve Bayes classifiers for the personality traits, each instance of the datasets used to train them would have to possess data from all of the six interaction features, meaning that there would have to be a correspondence between all the records. However, keyboard and mouse activities may not be simultaneous or happen with the same frequency, resulting in log datasets with different sizes. The solution for this problem was to calculate the mean values of the features for each individual every half hour, during the length of the experiment. Using this method, and after a selection of the valid records, it was possible to obtain a dataset with 138 instances with values of the individuals' key down time, time between keys, mouse velocity, mouse acceleration, the distance pointer to line between clicks and the absolute sum of degrees between clicks. Then, results of each individual's NPA questionnaire were added to this dataset. These results consist of the categories the subjects got for Openness,

\footnotetext{
${ }^{1}$ The following sensors have been used in the experiment:computer keyboard, mouse, temperature, luminosity, and sound sensors
} 
Conscientiousness, Extraversion, Agreeableness, Neuroticism and the final emotion in their personalities (as determined as described in Sec. 3.2).

The full set variables consisted of the following: $T B K, K D T, M V, M A, A S A, D P T L B C$, Openness, Conscientiousness, Extraversion, Agreableness, Neuroticism and Emotion.

Bayesian models do not perform well in continuous data. Therefore, it was necessary to discretize the values of the interaction features. Using Weka, and more specifically the PreProcess tab of the Explorer interface, a supervised filter called Discretize was applied do the dataset in order to convert these values into intervals [12]. In order to select the variables with the strongest dependence relationship, the dataset underwent an attribute selection phase, supported by the resources in the Select Attributes tab of the Explorer interface. The ClassifierAttributeEval was selected as the attribute evaluator. Accordingly, the Naïve Bayes was set as the target classifier and the parameter folds was set to 12 for 12 -fold cross validation. Only the three best features were selected for each personality trait. The results showed that the DPTLBC is always selected as one of the top three features, which reveals that the behaviour of this variable is more permeable to the characteristics of an individual's personality. On the other hand, the $K D T$ is never selected, which eventually means that it is the feature that reflects less the personality traits.

\subsection{Model Construction and Assessment}

The Naïve Bayes classifiers were learned using subsets of the dataset containing all the attributes. The learning phase was executed using $R$ software, more specifically the functionalities provided by the bnlearn library. 12-fold cross validation was performed in order to assess the model with the classification error [12] as the loss function. In the attribute selection phase, the top three features for Neurotiscism and Emotion were $D P T L B C, M V, A S A$ and $D P T L B C, M V, M A$, respectively. The mean classification errors for the classifiers using these features were 0.08018 (for Neuroticism) and 0.09596 (for Emotion). The resulting model is depicted in the following diagram 1.

The Neuroticism and Emotion classifiers are, eventually, the most important. Neuroticism is a personality trait characterized by anxiety, moodiness and worry. The individuals that score high on Neuroticism usually respond poorly to stressors and manifest a change in behaviour when submitted to them. The Neuroticism categories present in the model has high, low and medium low. By conditioning the features in the Neuroticism classifier, it is possible to detect significant changes in its behaviour. In the Emotion classifier, the analyzed categories were anxious, bored and relaxed. When looking at it and performing the same type of conditioning for the features mouse velocity, mouse acceleration and the distance of the pointer to the line between each two consecutive clicks, it is revealed that the anxious class has higher probabilities for the central intervals of these features. For low intervals of DPTLBC, $M V$ and $A S A$ it is possible to see that the classifier assigns higher probabilities to the high class of Neuroticism. This indicates that more precision may be associated with high score in Neuroticism. This is also in line to what is expect as a tendency for the evolution of an individual in a stressed state [11]. The emotions expressed in the upper and lower boundaries of the features are usually bored and relaxed. 


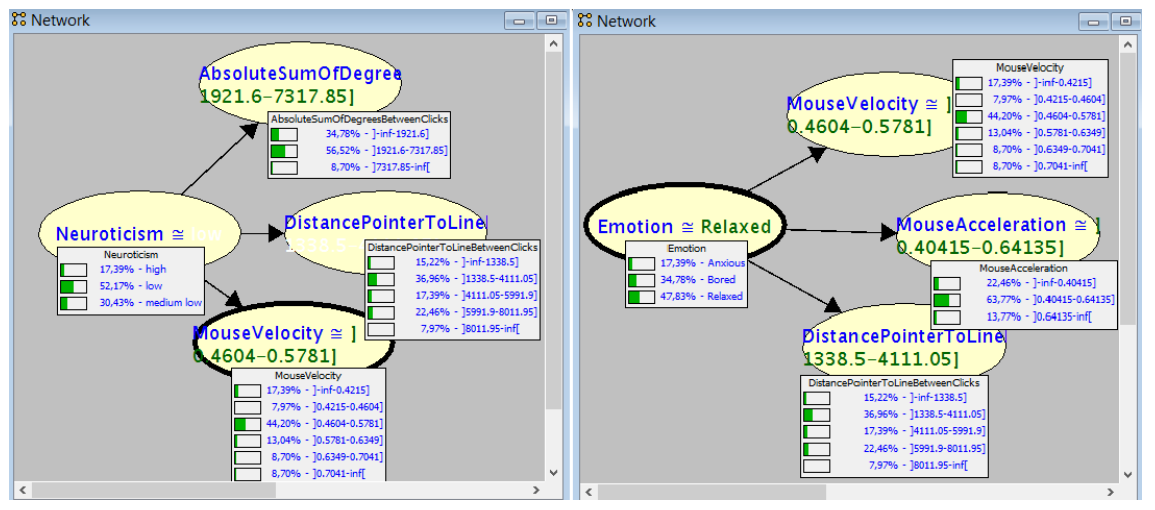

Fig. 1. The resulting Bayes model for the Neuroticism and Emotion networks.

\section{Conclusions}

In order to develop an intelligent system that supports human communication in regular or demanding circumstances, a computational support is required that describes the richness of a human's emotional state in relation to communication and the environment. To this end, this paper presented significant advantages for understating how psychological and stress dimensions can be related in certain circumstances and how they can be identified using everyday devices.

Using a non-intrusive approach an experiment under different parameter settings have been performed. This experiment pointed dependence relationships between the features being monitored and the main personality traits. These relationships, especially those regarding Neuroticism, have the potential to enable the characterization of individuals through their device interaction patterns. The identification of a neurotic personality is an indicator that a person may not deal well with potentially stressful situations, which may impair their communication capabilities.

The findings presented herein may help to identify the degree of relationship between one's personality characteristics and his current stress state, maximizing the benefits of the communication within virtual environment applications in fields such as learning, medicine or conflict resolution. The main contribution of this work is in the identification of situations in which actions are required in order to smooth processes and not let people loose efficiency when they get ensnared by their own circumstances.

\section{Acknowledgements}

This work was developed in the context of the project CAMCoF - Context-aware Multimodal Communication Framework funded by ERDF - European Regional Development Fund through the COMPETE Programme (operational programme for competitiveness) and by National Funds through the FCT - Fundação para a Ciência e a Tecnologia 
(Portuguese Foundation for Science and Technology) within project FCOMP-01-0124FEDER-028980.

\section{References}

1. Bell, M.: Toward a definition of virtual worlds. Journal For Virtual Worlds Research 1(1) (2008), http://journals.tdl.org/jvwr/index.php/jvwr/article/view/283

2. Carneiro, D., Castillo, J.C., Novais, P., Fernández-Caballero, A., Neves, J.: Multimodal behavioral analysis for non-invasive stress detection. Expert Syst. Appl. 39(18), 13376-13389 (Dec 2012), http://dx.doi.org/10.1016/j.eswa.2012.05.065

3. Costa, P., MacCrae, R., Psychological Assessment Resources, I.: Revised NEO Personality Inventory (NEO PI-R) and NEO Five-Factor Inventory (NEO FFI): Professional Manual. Psychological Assessment Resources (1992), http://books.google.pt/books?id=mp3zNwAACAAJ

4. Friedman, N., Geiger, D., Goldszmidt, M.: Bayesian network classifiers. Machine learning 29(2-3), 131-163 (1997)

5. Gebhard, P.: ALMA: a layered model of affect. Proceedings of the fourth international joint conference on Autonomous agents and multiagent systems pp. 29-36 (2005)

6. Jain, A., Bolle, R., Pankanti, S.: Introduction to biometrics. In: Jain, A., Bolle, R., Pankanti, S. (eds.) Biometrics, pp. 1-41. Springer US (1996), http://dx.doi.org/10.1007/0-306-470446_1

7. Jensen, F.V.: Bayesian networks. Wiley Interdisciplinary Reviews: Computational Statistics 1(3), 307-315 (2009)

8. Nettle, D.: Personality: What Makes You the Way You are. OUP Oxford (2007), http://books.google.pt/books?id=vk1kXARv1-IC

9. Ortony, A., Clore, G., Collins, A.: Cognitive Structure of Emotions. Cambridge University Press (1988)

10. Parsons, K.: Environmental ergonomics: a review of principles, methods and models. Applied Ergonomics 31(6), 581-594 (Dec 2000), http://dx.doi.org/10.1016/s00036870(00)00044-2

11. Rodrigues, M., Gonçalves, S., Carneiro, D., Novais, P., Fdez-Riverola, F.: Keystrokes and clicks: Measuring stress on e-learning students. In: Casillas, J., Martnez-Lpez, F.J., Vicari, R., De la Prieta, F. (eds.) Management Intelligent Systems, Advances in Intelligent Systems and Computing, vol. 220, pp. 119-126. Springer International Publishing (2013), http://dx.doi.org/10.1007/978-3-319-00569-0_15

12. Witten, I., Frank, E., Hall, M.: Data Mining: Practical machine learning tools and techniques. Morgan Kaufmann, third edn. (2011)

13. Yampolskiy, R.V., Govindaraju, V.: Behavioural biometrics: a survey and classification. International Journal of Biometrics 1(1), 81 (2008), http://www.inderscience.com/link.php?id=18665 